\title{
Prolonged Use of Methyldopa in Severe Hypertension in Pregnancy
}

\author{
PRISCILLA KINCAID-SMITH,* M.B., B.SC., M.R.C.P., M.R.A.C.P., D.C.P. \\ MARGARET BULLEN, $\dagger$ M.A., M.B.; JEANNINE MILLS, $\dagger$ M.B., B.SC.
}

Brit. med. F., 1966, 1, 274-276

Although methyldopa has been widely used as a hypotensive drug for several years, little has been written about its use during pregnancy. Hans and Kopelman (1964) used this drug for short periods in pre-eclamptic toxaemia with apparent benefit.

Treatment with hypotensive drugs may be imperative for the safety of the mother when manifestations such as retinal haemorrhages develop during pregnancy. Even in malignant hypertension a successful pregnancy is possible when the blood-pressure is controlled (Kincaid-Smith et al., 1958). If there is no clear-cut indication that the mother requires treatment, interest centres around the possibility that hypotensive drugs may improve the outlook for the foetus. Presumably the foetus and the placental circulation derive the maximum benefit from treatment begun early in the pregnancy. In this paper we report our experience with the long-term use of methyldopa in hypertensive pregnant women.

\section{Clinical Material and Method of Treatment}

Thirty-two patients with severe hypertension were treated with methyldopa during pregnancy. The details are given in Table II. Three patients had had blood-pressure readings taken only during pregnancy; the remainder were known to be hypertensive when they were not pregnant (Table II). Twentyfive patients had had previous pregnancies, all but two having been hypertensive during pregnancy, and 15 of them had had previous unsuccessful pregnancies.

Even before treatment was begun the blood-pressure level during pregnancy was usually lower than that recorded before or after the pregnancy (Table II). The patients were a heterogeneous group and about a third had known or presumed underlying renal disease. We did not include those with preeclamptic toxaemia alone, but in 12 patients pre-eclamptic toxaemia probably developed as a complication.

Two patients were treated throughout the whole pregnancy, and most were treated for periods of three to six months. In five the drug was used in the first trimester (Table II).

Methyldopa was the only hypotensive drug used in most patients, but seven also received reserpine or chlorothiazide at some time during the pregnancy. The initial dose of methyldopa was usually $0.25 \mathrm{~g}$. daily, and this was increased in an attempt to maintain the blood-pressure at $140 / 90$ or lower in the standing position. The final total daily doses ranged from 0.5 to $4 \mathrm{~g}$.

\section{Results of Treatment}

Table II shows the details of treatment and the outcome of the pregnancy. The blood-pressure was usually satisfactorily controlled. The difference between the blood-pressures immediately before treatment was started and those taken at the midpoint of treatment were highly significant (Table I).

\footnotetext{
* Honorary Physician, Queen Victoria Hospital, Melbourne; Senior Associate in Medicine, University of Melbourne. In receipt of grant from the National Health and Medical Research Council of Australia.

† Research Assistant.
}

TABLE I.-Difference in Blood-pressures Immediately Before Treatment and Those Taken at the Mid-point of Treatment

\begin{tabular}{|c|c|c|c|}
\hline & & Systolic B.P. & Diastolic B.P. \\
\hline $\begin{array}{l}\text { Number of patients } \\
\text { Mean difference } \\
\text { Standard error }\end{array}$ & $\begin{array}{ll} & \cdots \\
\cdots & \cdots\end{array}$ & $\begin{array}{l}29 \\
36 \cdot 6 \\
3 \cdot 33\end{array}$ & $\begin{array}{l}29 \\
24 \cdot 7 \\
2 \cdot 38\end{array}$ \\
\hline
\end{tabular}

So far as was possible treatment was carried out on an outpatient basis and patients were admitted to hospital only if the blood-pressure was difficult to control or if they developed other abnormalities such as oedema or proteinuria.

Foetal Mortality and Morbidity.-The babies were examined by members of the obstetric staff of the hospital, and premature babies or those with any apparent abnormality were seen by a paediatrician. No significant abnormalities were noted in this group. Four babies had umbilical herniae, and one was noted to have slight clitoral enlargement. One baby who had cyanotic attacks had a systolic cardiac murmur, which after cardiological investigation was stated to be functional. Two patients who developed superimposed pre-eclamptic toxaemia before the 30th week lost their babies; one of these was stillborn and the other died after a few hours (foetal weight 700 g.). One patient, who habitually lost her baby at about the same stage of pregnancy, had a foetal death in utero after premature rupture of the membranes at 27 weeks.

Pre-eclamptic Toxaemia.-It may be very difficult to assess the onset of pre-eclamptic toxaemia in a group of patients who are already hypertensive and some of whom have proteinuria. However, in 12 patients we made a diagnosis of pre-eclamptic toxaemia on the basis of an increase in or appearance of proteinuria or oedema or because of a sudden increase in hypertension. The serum uric-acid level was sometimes of value in confirming this diagnosis (Table II). We did not obtain any clear-cut improvement in the pre-eclamptic toxaemia as a result of methyldopa treatment in these patients. Bed rest, together with an increased dose of methyldopa, controlled the hypertension and at the same time the proteinuria sometimes decreased or disappeared. However, the persistence of a high serum uric acid, retinal arterial narrowing, and, in some instances, proteinuria seemed to indicate that lowering the blood-pressure with methyldopa did not counteract the basic abnormality of pre-elamptic toxaemia.

Side-effects and Withdrawal of Methyldopa.-No serious side-effects were encountered, but several patients complained of drowsiness. Methyldopa was discontinued in one patient at 29 weeks because the blood-pressure had fallen too low. This patient had been on reserpine and chlorothiazide before the pregnancy, and methyldopa had been added to achieve better control of the blood-pressure (Case 10).

\section{Discussion}

Our main purpose in reporting our experience with methyldopa is to record that it is an effective hypotensive agent in pregnancy hypertension. There is probably some advantage in treating subjects as out-patients, because methyldopa is relatively more effective when the patient is in the upright position. The freedom from side-effects or severe postural hypo- 


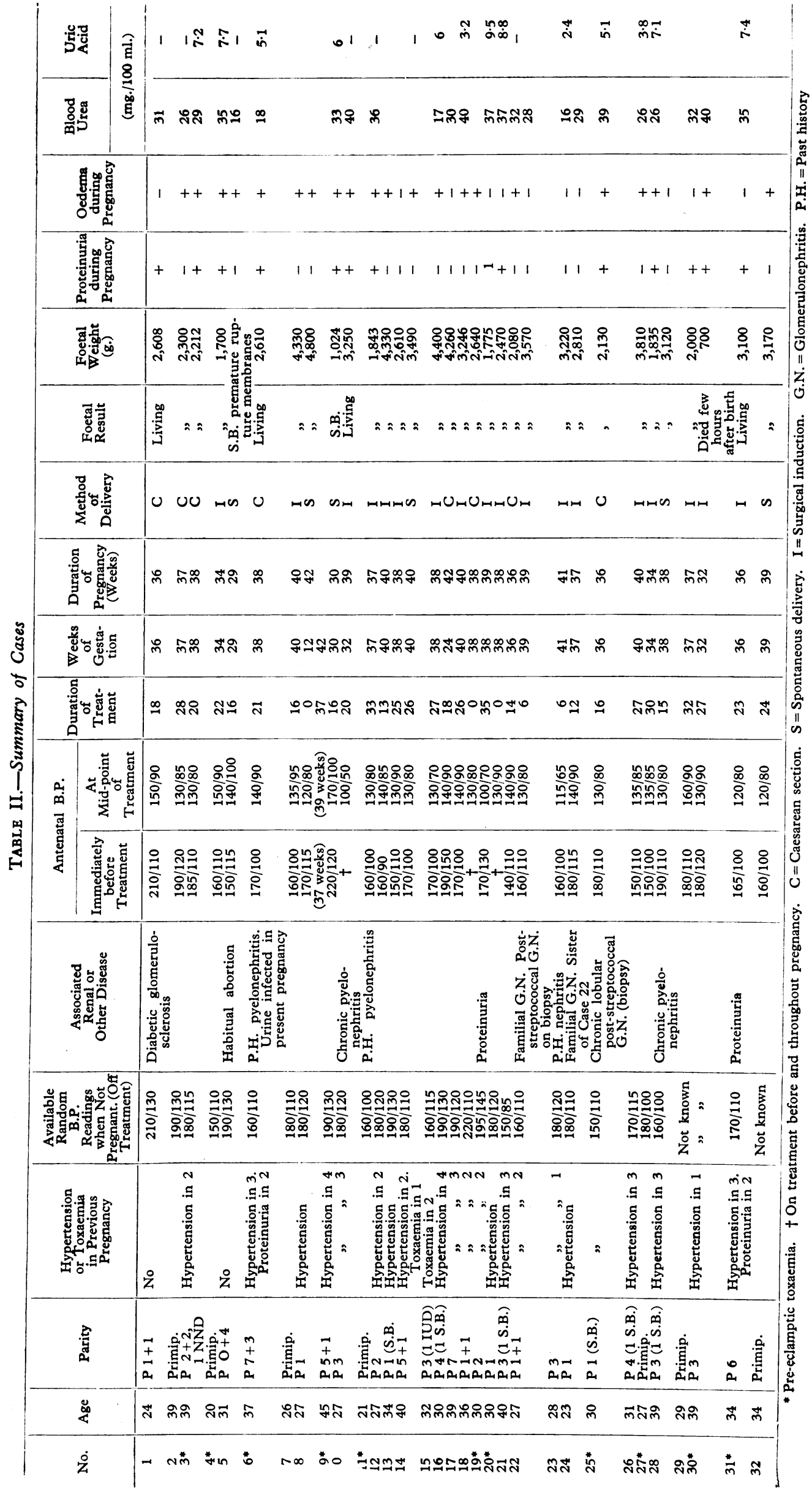


tension permitted easy regulation of the blood-pressure in outpatients.

Since the thalidomide disasters both doctors and mothers are understandably hesistant about the use of a new drug during pregnancy. It is therefore reassuring that we found no evidence of ill effects on the foetus in patients treated in the first trimester or with doses of methyldopa up to $4 \mathrm{~g}$. daily.

The perinatal mortality rate of $9.3 \%$ does not seem excessive for this group of severely hypertensive women, particularly as one of the three foetal deaths was thought to be unrelated to the hypertensive disease, which reduces this figure to $6.2 \%$. Townsend (1958), studying a similar population group in Melbourne, found a perinatal mortality of $16 \%$ when mothers had an initial diastolic pressure of $110 \mathrm{~mm} . \mathrm{Hg}$, and this figure rose to $50 \%$ in patients in whom this degree of hypertension was complicated by proteinuria. Twelve of our patients had proteinuria, including the two who lost their babies as a result of developing pre-eclamptic toxaemia before the thirtieth week.

As MacGillivray (1964) has pointed out, only a careful controlled trial will establish whether hypotensive drugs increase the rate of successful pregnancies in hypertensive mothers.

We are now conducting a controlled trial of methyldopa in pregnancy hypertension because we feel that it has definite practical advantages as well as the possible theoretical advantage of inhibition of decarboxylase (Brit. med. F., 1964).

\section{Summary}

Methyldopa has been used to control hypertension in pregnancy for periods up to the whole gestation time without any apparent adverse effect on the foetus.

The blood-pressure can usually be easily controlled on an out-patient basis and side-effects were minimal.

Only 3 out of 32 severely hypertensive women lost their babies, and as one foetal death was due to premature rupture of the membranes the corrected perinatal mortality rate was only $6.2 \%$.

We are grateful to the obstetric staff of the Queen Victoria Hospital for referring their patients for treatment, and to Merck Sharp and Dohme (Australia) Limited and Dr. D. C. Mezey, Director of International Clinical Research, for supplies of Aldomet for this study. Professor R. R. H. Lovell gave helpful advice about the analysis of the blood-pressure findings, and the statistical analyses were carried out by Miss B. Laby, of the University of Melbourne, Department of Statistics.

\section{REFERENCES}

Brit. med. F., 1964, 2, 459.

Hans, S. F., and Kopelman, H. (1964). Brit. med. Y., 1, 736. Kincaid-Smith, P., Sommers, K., and McClure Browne, J. C. (1958).

MacGillivray, I. (1964). Brit. med. f., 1, 978.

Townsend, S. L. (1958). Thesis for Doctor of Medicine. University of Melbourne.

\title{
Trial of Ambilhar, a Nitrothiazole Derivative, in $S$. mansoni
}

\author{
P. JORDAN,* M.D., D.T.M.\&H.
}

Brit. med. F., 1966, 1, 276-278

The schistosomicidal action of Ambilhart (a nitrothiazole derivative, 1-(5-nitro-2-thiazolyl)-2-imidazolidinone) in mice infected with Schistosoma mansoni was reported by Lambert (1964). Preliminary observations of its use in man were recorded by Jordan (1966a), and the present communication reports parasitological findings obtained four months after treatment.

\section{Methods and Materials}

The use of the percentage reduction in egg load was suggested by Jordan and Randall (1962) for assessing results of suppressive therapy in Schistosoma haematobium infections, and Bradley (1963) showed that the "cure rate" in this infection was inversely proportional to the initial egg output. Bell (1964) outlined the advantages of using the former parameter of chemotherapeutic activity of schistosomicidal drugs and devised a new method for the necessary quantitative studies in relation to $S$. mansoni infections (Bell, 1963). This method was used in the present trials. Bell (1964) stressed that a $100 \%$ reduction in egg load (by the filtration-staining technique) does not in fact imply a cure, but merely an egg output of less than 1,000 eggs a day. Within this context "cure rates" are given for results obtained by the filtration-staining technique. On the other hand, this method, combined with further stool examinations and the use of other techniques, more closely approaches what may be necessary to evaluate a true parasitological cure, and when three techniques have been used and three or four

* East African Institute for Medical Research, Mwanza, Tanzania. + Formerly known as Ciba 32,644-Ba. stools examined the absence of evidence of $S$. mansoni is considered for the purpose of this communication to represent a cure.

Children and adults excreting eggs of $S$. mansoni were treated in these trials. The former were found infected in school surveys ; the latter requested treatment-invariably complaining of abdominal pain-and on investigation were found to be infected. All patients were treated as in-patients with a dose of $25 \mathrm{mg} . / \mathrm{kg}$. body-weight daily (in two doses) for five days.

Prior to treatment patients were given a general clinical examination, and a 24-hour collection of stool was made for processing by the filtration-staining technique (Bell, 1963) in order to assess the 24-hour output of $S$. mansoni eggs and to give an indication of the intensity of infection.

At follow-up examination two and four months after treatment, patients were readmitted for the collection of a further 24-hour stool for quantitative determination of the egg load, and an additional stool was collected; three 1-g. aliquots were examined for $S$. mansoni eggs by the A.M.S. III technique (Hunter et al., 1948), the rest of the stool being treated for hatching of eggs and examination for miracidia. Thus at the follow-up examinations three or four stools were generally examined and three different techniques were used for the detection of eggs or miracidia.

\section{Results}

Comparative results of the quantitative filtration-staining technique two and four months after treatment were obtained 